\title{
A new class of Hermite-Fubini polynomials and its properties
}

\section{Waseem A. Khan ${ }^{1}$ and Nisar K S ${ }^{2}$}

${ }^{1}$ Department of Mathematics, Faculty of Science, Integral University, Lucknow-226026, (India)

${ }^{2}$ Department of Mathematics, College of Arts and Science-Wadi Al dawaser, Prince Sattam bin Abdulaziz University, Riyadh region 11991, Saudi Arabia

E-mail: waseem08_khan@rediffmail.com,n.sooppy@psau.edu.sa

\begin{abstract}
In this paper, we introduce a new class of Hermite-Fubini numbers and polynomials and investigate some properties of these polynomials. We establish summation formulas of these polynomials by summation techniques series. Furthermore, we derive symmetric identities of Hermite-Fubini numbers and polynomials by using generating functions.
\end{abstract}

Keywords: Hermite polynomials, Fubini numbers and polynomials, Hermite-Fubini polynomials, summation formulae, symmetric identities.

2010 Mathematics Subject Classification.: 11B68, 11B75, 11B83, 33C45, 33C99.

\section{Introduction}

As is well known, the 2-variable Hermite Kampé de Fériet polynomials (2VHKdFP) $H_{n}(x, y)[1,3]$ are defined as

$$
H_{n}(x, y)=n ! \sum_{r=0}^{\left[\frac{n}{2}\right]} \frac{y^{r} x^{n-2 r}}{r !(n-2 r) !} .
$$

It is clear that

$$
H_{n}(2 x,-1)=H_{n}\left(x, H_{n}\left(x,-\frac{1}{2}\right)=H e_{n}(x), H_{n}(x, 0)=x^{n},\right.
$$

where $H_{n}(x)$ and $H_{n}(x)$ being ordinary Hermite polynomials.

The Hermite polynomial $H_{n}(\mathrm{x}, \mathrm{y})$ (see $([9,10])$ is defined by means of the following generating function as follows:

$$
e^{x t+y t^{2}}=\sum_{n=0}^{\infty} H_{n}(x, y) \frac{t^{n}}{n !}
$$

Geometric polynomials (also known as Fubini polynomials) are defined as follows (see [2]):

$$
F_{n}(x)=\sum_{k=0}^{n}\left\{\begin{array}{l}
n \\
k
\end{array}\right\} k ! x^{k}
$$

where $\left\{\begin{array}{c}n \\ k\end{array}\right\}$ is the Stirling number of the second kind (see [5]).

For $x=1$ in (1.3), we get $n^{\text {th }}$ Fubini number (ordered Bell number or geometric number) $F_{n}[2,4,5,6,8,12]$ is defined by

$$
F_{n}(1)=F_{n}=\sum_{k=0}^{n}\left\{\begin{array}{l}
n \\
k
\end{array}\right\} k !
$$


The exponential generating functions of geometric polynomials is given by (see [2]):

$$
\frac{1}{1-x\left(e^{t}-1\right)}=\sum_{n=0}^{\infty} F_{n}(x) \frac{t^{n}}{n !}
$$

and related to the geometric series (see [2]):

$$
\left(x \frac{d}{d x}\right)^{m} \frac{1}{1-x}=\sum_{k=0}^{\infty} k^{m} x^{k}=\frac{1}{1-x} F_{m}\left(\frac{x}{1-x}\right),|x|<1 .
$$

Let us give a short list of these polynomials and numbers as follows:

$F_{0}(x)=1, F_{1}(x)=x, F_{2}(x)=x+2 x^{2}, F_{3}(x)=x+6 x^{2}+6 x^{3}, F_{4}(x)=x+14 x^{2}+36 x^{3}+24 x^{4}$,

and

$$
F_{0}=1, F_{1}=1, F_{2}=3, F_{3}=13, F_{4}=75 .
$$

Geometric and exponential polynomials are connected by the relation (see [2]):

$$
F_{n}(x)=\int_{0}^{\infty} \phi_{n}(x) e^{-\lambda} d \lambda
$$

Recently, Pathan and Khan [9] introduced two variable Hermite-Bernoulli polynomials is defined by means of the following generating function:

$$
\left(\frac{t}{e^{t}-1}\right)^{\alpha} e^{x t+y t^{2}}=\sum_{n=0}^{\infty} H_{n}^{(\alpha)}(x, y) \frac{t^{n}}{n !} .
$$

On setting $\alpha=1$ in (1.7), the result reduces to known result of Dattoli et al. $[3]$.

The manuscript of this paper as follows: In section 2, we consider generating functions for Hermite-Fubini numbers and polynomials and give some properties of these numbers and polynomials. In section 3, we derive summation formulas of Hermite-Fubini numbers and polynomials. In Section 4, we construct a symmetric identities of Hermite-Fubini numbers and polynomials by using generating functions.

\section{A new class of Hermite-Fubini numbers and polynomials}

In this section, we define three-variable Hermite-Fubini polynomials and obtain some basic properties which gives us new formula for ${ }_{H} F_{n}(x, y, z)$. Moreover, we shall consider the sum of products of two Hermite-Fubini polynomials. The sum of products of various polynomials and numbers with or without binomial coefficients have been studied by (see $[2,4,5,6,8])$ :

We introduce 3-variable Hermite-Fubini polynomials by means of the following generating function:

$$
\frac{e^{x t+y t^{2}}}{1-z\left(e^{t}-1\right)}=\sum_{n=0}^{\infty}{ }_{H} F_{n}(x, y ; z) \frac{t^{n}}{n !} .
$$

It is easily seen from definition (2.1), we have

$$
{ }_{H} F_{n}(0,0 ; z)=F_{n}(z),{ }_{H} F_{n}(0,0 ; 1)=F_{n} .
$$

For $y=0$ in (2.1), we obtain 2-variable Fubini polynomials which is defined by Kargin [8]. 


$$
\frac{e^{x t}}{1-z\left(e^{t}-1\right)}=\sum_{n=0}^{\infty} F_{n}(x ; z) \frac{t^{n}}{n !} .
$$

When investigating the connection between Hermite polynomials $H_{n}(x, y)$ and Fubini polynomials $F_{n}(z)$ of importance is the following theorem.

Theorem 2.1. The following summation formula for Hermite-Fubini polynomials holds true:

$$
\begin{gathered}
\frac{e^{-y t^{2}}}{\Omega}[\cos x t(z+1)-z \cos (t-x t)]=\sum_{n=0}^{\infty} H_{H} F_{2 n}(x, y, z) \frac{(-1)^{n} t^{2 n}}{(2 n) !} \\
\frac{e^{-y t^{2}}}{\Omega}[\sin x t(z+1)+z \sin (t-x t)]=\sum_{n=0}^{\infty}{ }_{H} F_{2 n+1}(x, y, z) \frac{(-1)^{n} t^{2 n+1}}{(2 n+1) !},
\end{gathered}
$$

where $\Omega=[1-z(\cos t-1)]^{2}+[z \sin t]^{2}$.

Proof. On separating the power series on r.h.s. of (2.1) in to their even and odd terms by using the elementary identity

$$
\sum_{n=0}^{\infty} f(n)=\sum_{n=0}^{\infty} f(2 n)+\sum_{n=0}^{\infty} f(2 n+1)
$$

and then replacing $t$ by $i t$ where $i^{2}=-1$ and equating the real and imaginary parts in the resulting equation, we get the summation formulae (2.2) and (2.3).

Remark 2.1. On setting $x=y=0, z=1$ in (2.3) and (2.4), we get the following well-known classical results involving Fubini numebrs.

Corollary 2.1. The following summation formula for Hermite-Fubini polynomials holds true:

$$
\begin{gathered}
\frac{2-\cos t}{5-4 \cos t}=\sum_{n=0}^{\infty} F_{2 n} \frac{(-1)^{n} t^{2 n}}{(2 n) !} \\
\frac{\sin t}{5-4 \cos t}=\sum_{n=0}^{\infty} F_{2 n+1} \frac{(-1)^{n} t^{2 n+1}}{(2 n+1) !} .
\end{gathered}
$$

Theorem 2.2. For $n \geq 0$, the following formula for Hermite-Fubini polynomials holds true:

$$
{ }_{H} F_{n}(x, y, z)=\sum_{m=0}^{n}\left(\begin{array}{l}
n \\
r
\end{array}\right) F_{n-m}(z) H_{m}(x, y) .
$$

Proof. Using definition (2.1), we have

$$
\begin{gathered}
\sum_{n=0}^{\infty}{ }_{H} F_{n}(x, y, z) \frac{t^{n}}{n !}=\frac{e^{x t+y t^{2}}}{1-z\left(e^{t}-1\right)} \\
=\sum_{n=0}^{\infty} F_{n}(z) \frac{t^{n}}{n !} \sum_{m=0}^{\infty} H_{m}(x, y) \frac{t^{m}}{m !} \\
=\sum_{n=0}^{\infty}\left(\sum_{m=0}^{n}\left(\begin{array}{c}
n \\
r
\end{array}\right) F_{n-m}(z) H_{m}(x, y)\right) \frac{t^{n}}{n !} .
\end{gathered}
$$

Comparing the coefficients of $\frac{t^{n}}{n !}$ yields $(2.7)$. 
Theorem 2.2. For $n \geq 0$, the following formula for Hermite-Fubini polynomials holds true:

$$
H_{n}(x, y)={ }_{H} F_{n}(x, y ; z)-z_{H} F_{n}(x+1, y ; z)+z_{H} F_{n}(x, y ; z) .
$$

Proof. We begin with the definition (2.1) and write

$$
\begin{gathered}
e^{x t+y t^{2}}=\frac{1-z\left(e^{t}-1\right)}{1-z\left(e^{t}-1\right)} e^{x t+y t^{2}} \\
=\frac{e^{x t+y t^{2}}}{1-z\left(e^{t}-1\right)}-\frac{z\left(e^{t}-1\right)}{1-z\left(e^{t}-1\right)} e^{x t+y t^{2}}
\end{gathered}
$$

Then using the definition of Kampé de Fériet generalization of the Hermite polynomials $H_{n}(x, y)$ and $(2.1)$, we have

$$
\sum_{n=0}^{\infty} H_{n}(x, y) \frac{t^{n}}{n !}=\sum_{n=0}^{\infty}\left[{ }_{H} F_{n}(x, y ; z)-z_{H} F_{n}(x+1, y ; z)+z_{H} F_{n}(x, y ; z)\right] \frac{t^{n}}{n !} .
$$

Finally, comparing the coefficients of $\frac{t^{n}}{n !}$, we get (2.8).

Theorem 2.3. For $n \geq 0$ and $z_{1} \neq z_{2}$, the following formula for Hermite-Fubini polynomials holds true:

$$
\begin{gathered}
\sum_{k=0}^{n}\left(\begin{array}{c}
n \\
k
\end{array}\right){ }_{H} F_{n-k}\left(x_{1}, y_{1} ; z_{1}\right)_{H} F_{k}\left(x_{2}, y_{2} ; z_{2}\right) \\
=\frac{z_{2 H} F_{n}\left(x_{1}+x_{2}, y_{1}+y_{2} ; z_{2}\right)-z_{1 H} F_{n}\left(x_{1}+x_{2}, y_{1}+y_{2} ; z_{1}\right)}{z_{2}-z_{1}} .
\end{gathered}
$$

Proof. The products of $(2.1)$ can be written as

$$
\begin{gathered}
\sum_{n=0}^{\infty} \sum_{k=0}^{\infty}{ }_{H} F_{n}\left(x_{1}, y_{1} ; z_{1}\right) \frac{t^{n}}{n !}{ }_{H} F_{k}\left(x_{2}, y_{2} ; z_{2}\right) \frac{t^{k}}{k !}=\frac{e^{x_{1} t+y_{1} t^{2}}}{1-z_{1}\left(e^{t}-1\right)} \frac{e^{x_{2} t+y_{2} t^{2}}}{1-z_{2}\left(e^{t}-1\right)} \\
\sum_{n=0}^{\infty}\left(\sum_{k=0}^{n}\left(\begin{array}{c}
n \\
k
\end{array}\right){ }_{H} F_{n-k}\left(x_{1}, y_{1} ; z_{1}\right)_{H} F_{k}\left(x_{2}, y_{2} ; z_{2}\right)\right) \frac{t^{n}}{n !} \\
=\frac{z_{2}}{z_{2}-z_{1}} \frac{e^{\left(x_{1}+x_{2}\right) t+\left(y_{1}+y_{2}\right) t^{2}}}{1-z_{1}\left(e^{t}-1\right)}-\frac{z_{1}}{z_{2}-z_{1}} \frac{e^{\left(x_{1}+x_{2}\right) t+\left(y_{1}+y_{2}\right) t^{2}}}{1-z_{2}\left(e^{t}-1\right)} \\
=\left(\frac{z_{2 H} F_{n}\left(x_{1}+x_{2}, y_{1}+y_{2} ; z_{2}\right)-z_{1 H} F_{n}\left(x_{1}+x_{2}, y_{1}+y_{2} ; z_{1}\right)}{z_{2}-z_{1}}\right) \frac{t^{n}}{n !}
\end{gathered}
$$

By equating the coefficients of $\frac{t^{n}}{n !}$ on both sides, we get (2.9).

Theorem 2.4. For $n \geq 0$, the following formula for Hermite-Fubini polynomials holds true:

$$
z_{H} F_{n}(x+1, y ; z)=(1+z)_{H} F_{n}(x, y ; z)-H_{n}(x, y) \text {. }
$$

Proof. From (2.1), we have

$$
\begin{gathered}
\sum_{n=0}^{\infty}\left[{ }_{H} F_{n}(x+1, y ; z)-{ }_{H} F_{n}(x, y ; z)\right] \frac{t^{n}}{n !}=\frac{e^{x t+y t^{2}}}{1-z\left(e^{t}-1\right)}\left(e^{t}-1\right) \\
=\frac{1}{z}\left[\frac{e^{x t+y t^{2}}}{1-z\left(e^{t}-1\right)}-e^{x t+y t^{2}}\right] \\
=\frac{1}{z} \sum_{n=0}^{\infty}\left[{ }_{H} F_{n}(x, y ; z)-H_{n}(x, y)\right] \frac{t^{n}}{n !}
\end{gathered}
$$


Comparing the coefficients of $\frac{t^{n}}{n !}$ on both sides, we obtain (2.10).

Remark 2.3. On setting $x=y=0$ and $x=-1$ in Theorem 2.4, we find

$$
z_{H} F_{n}(1,0 ; z)=(1+z)_{H} F_{n}(0,0 ; z)
$$

and

$$
z_{H} F_{n}(0,0 ; z)=(1+z)_{H} F_{n}(-1,0 ; z)-(-1)^{n}
$$

Theorem 2.5. For $n \geq 0, p, q \in \mathbb{R}$, the following formula for Hermite-Fubini polynomials holds true:

$$
{ }_{H} F_{n}(p x, q y ; z)=n ! \sum_{k=0}^{n} \sum_{j=0}^{\left[\frac{k}{2}\right]}{ }_{H} F_{n-k}(x, y ; z)((p-1) x)^{k}((q-1) y)^{j} \frac{1}{(n-k-2 j) ! j !} .
$$

Proof. Rewrite the generating function (2.1), we have

$$
\begin{aligned}
& \sum_{n=0}^{\infty}{ }_{H} F_{n}(p x, q y ; z) \frac{t^{n}}{n !}=\frac{1}{1-z\left(e^{t}-1\right)} e^{x t+y t^{2}} e^{(p-1) x t} e^{(q-1) y t^{2}} \\
= & \left(\sum_{n=0}^{\infty} H F_{n}(x, y ; z) \frac{t^{n}}{n !}\right)\left(\sum_{k=0}^{\infty}((p-1) x)^{k} \frac{t^{k}}{k !}\right)\left(\sum_{j=0}^{\infty}((q-1) y)^{j} \frac{t^{2 j}}{j !}\right) \\
= & \left(\sum_{n=0}^{\infty} H F_{n}(x, y ; z) \frac{t^{n}}{n !}\right)\left(\sum_{k=0}^{\infty} \sum_{j=0}^{\infty}((p-1) x)^{k}((q-1) y)^{j} \frac{t^{k+2 j}}{n ! k ! j !}\right)
\end{aligned}
$$

Replacing $k$ by $k-2 j$ in above equation, we have

$$
\begin{aligned}
\text { L.H.S. } & =\left(\sum_{n=0}^{\infty}{ }_{H} F_{n}(x, y ; z) \frac{t^{n}}{n !}\right)\left(\sum_{k=2 j}^{\infty}((p-1) x)^{k-2 j}((q-1) y)^{j} \frac{t^{k}}{(k-2 j) ! j !}\right) \\
& =\sum_{n=0}^{\infty} \sum_{k=2 j}^{\infty} H_{n} F_{n}(x, y ; z)((p-1) x)^{k-2 j}((q-1) y)^{j} \frac{t^{n+k}}{(k-2 j) ! j ! n !}
\end{aligned}
$$

Again replacing $n$ by $n-k$ in above equation, we have

$$
\text { L.H.S. }=\sum_{n=0}^{\infty} \sum_{k=0}^{n} \sum_{j=0}^{\left[\frac{k}{2}\right]}{ }_{H} F_{n-k}(x, y ; z)((p-1) x)^{k-2 j}((q-1) y)^{j} \frac{t^{n}}{(n-k-2 j) ! j ! k !} .
$$

Finally, equating the coefficients of $t^{n}$ on both sides, we acquire the result (2.13).

Theorem 2.6. For $n \geq 0$, the following formula for Hermite-Fubini polynomials holds true:

$$
{ }_{H} F_{n}(x, y, z)=\sum_{l=0}^{n}\left(\begin{array}{c}
n \\
l
\end{array}\right) H_{n-l}(x, y) \sum_{k=0}^{l} z^{k} k ! S_{2}(l, k)
$$

Proof. From (2.1), we have

$$
\begin{gathered}
\sum_{n=0}^{\infty}{ }_{H} F_{n}(x, y, z) \frac{t^{n}}{n !}=\frac{e^{x t+y t^{2}}}{1-z\left(e^{t}-1\right)} \\
=e^{x t+y t^{2}} \sum_{k=0}^{\infty} z^{k}\left(e^{t}-1\right)^{k}=e^{x t+y t^{2}} \sum_{k=0}^{\infty} z^{k} \sum_{l=k}^{\infty} k ! S_{2}(l, k) \frac{t^{l}}{l !} \\
=\sum_{n=0}^{\infty} H_{n}(x, y) \frac{t^{n}}{n !} \sum_{l=0}^{\infty} z^{k} \sum_{k=0}^{l} k ! S_{2}(l, k) \frac{t^{l}}{l !}
\end{gathered}
$$


Replacing $n$ by $n-l$ in above equation, we get

$$
\sum_{n=0}^{\infty}{ }_{H} F_{n}(x, y, z) \frac{t^{n}}{n !}=\sum_{n=0}^{\infty}\left(\sum_{l=0}^{n}\left(\begin{array}{l}
n \\
l
\end{array}\right) H_{n-l}(x, y) \sum_{k=0}^{l} z^{k} k ! S_{2}(l, k)\right) \frac{t^{n}}{n !} .
$$

Comparing the coefficients of $\frac{t^{n}}{n !}$ in both sides, we get (2.14).

Theorem 2.7. For $n \geq 0$, the following formula for Hermite-Fubini polynomials holds true:

$$
{ }_{H} F_{n}(x+r, y, z)=\sum_{l=0}^{n}\left(\begin{array}{c}
n \\
l
\end{array}\right) H_{n-l}(x, y) \sum_{k=0}^{l} z^{k} k ! S_{2}(l+r, k+r) .
$$

Proof. Replacing $x$ by $x+r$ in (2.1), we have

$$
\begin{gathered}
\sum_{n=0}^{\infty}{ }_{H} F_{n}(x+r, y, z) \frac{t^{n}}{n !}=\frac{e^{(x+r) t+y t^{2}}}{1-z\left(e^{t}-1\right)} \\
=e^{x t+y t^{2}} e^{r t} \sum_{k=0}^{\infty} z^{k}\left(e^{t}-1\right)^{k}=e^{x t+y t^{2}} e^{r t} \sum_{k=0}^{\infty} z^{k} \sum_{l=k}^{\infty} k ! S_{2}(l, k) \frac{t^{l}}{l !} \\
=\sum_{n=0}^{\infty} H_{n}(x, y) \frac{t^{n}}{n !} \sum_{l=0}^{\infty} z^{k} \sum_{k=0}^{l} k ! S_{2}(l+r, k+r) \frac{t^{l}}{l !}
\end{gathered}
$$

Replacing $n$ by $n-l$ in above equation, we get

$$
\sum_{n=0}^{\infty} H_{n} F_{n}(x+r, y, z) \frac{t^{n}}{n !}=\sum_{n=0}^{\infty}\left(\sum_{l=0}^{n}\left(\begin{array}{c}
n \\
l
\end{array}\right) H_{n-l}(x, y) \sum_{k=0}^{l} z^{k} k ! S_{2}(l+r, k+r)\right) \frac{t^{n}}{n !} .
$$

Comparing the coefficients of $\frac{t^{n}}{n !}$ in both sides, we get (2.15).

\section{Summation Formulae for Hermite-Fubini polynomials}

First, we prove the following result involving the Hermite-Fubini polynomials ${ }_{H} F_{n}(x, y ; z)$ by using series rearrangement techniques and considered its special case:

Theorem 3.1. The following summation formula for Hermite-Fubini polynomials ${ }_{H} F_{n}(x, y ; z)$ holds true:

$$
{ }_{H} F_{q+l}(w, y ; z)=\sum_{n, p=0}^{q, l}\left(\begin{array}{l}
q \\
n
\end{array}\right)\left(\begin{array}{l}
l \\
p
\end{array}\right)(w-y)^{n+p}{ }_{H} F_{q+l-n-p}(x, y ; z) .
$$

Proof. Replacing $t$ by $t+u$ in (2.1) and then using the formula [11,p.52(2)]:

$$
\sum_{N=0}^{\infty} f(N) \frac{(x+y)^{N}}{N !}=\sum_{n, m=0}^{\infty} f(n+m) \frac{x^{n}}{n !} \frac{y^{m}}{m !}
$$

in the resultant equation, we find the following generating function for the HermiteFubini polynomials ${ }_{H} F_{n}(x, y ; z)$ :

$$
\frac{1}{1-z\left(e^{t+u}-1\right)} e^{y(t+u)^{2}}=e^{-x(t+u)} \sum_{q, l=0}^{\infty}{ }_{H} F_{q+l}(x, y ; z) \frac{t^{q}}{q !} \frac{u^{l}}{l !}
$$


Replacing $x$ by $w$ in the above equation and equating the resultant equation to the above equation, we find

$$
\exp ((w-x)(t+u)) \sum_{q, l=0}^{\infty}{ }_{H} F_{q+l}(x, y ; z) \frac{t^{q}}{q !} \frac{u^{l}}{l !}=\sum_{q, l=0}^{\infty}{ }_{H} F_{q+l}(w, y ; z) \frac{t^{q}}{q !} \frac{u^{l}}{l !} .
$$

On expanding exponential function (3.4) gives

$$
\sum_{N=0}^{\infty} \frac{[(w-x)(t+u)]^{N}}{N !} \sum_{q, l=0}^{\infty}{ }_{H} F_{q+l}(x, y ; z) \frac{t^{q}}{q !} \frac{u^{l}}{l !}=\sum_{q, l=0}^{\infty}{ }_{H} F_{q+l}(w, y ; z) \frac{t^{q}}{q !} \frac{u^{l}}{l !}
$$

which on using formula (3.2) in the first summation on the left hand side becomes

$$
\sum_{n, p=0}^{\infty} \frac{(w-x)^{n+p} t^{n} u^{p}}{n ! p !} \sum_{q, l=0}^{\infty}{ }_{H} F_{q+l}(x, y ; z) \frac{t^{q}}{q !} \frac{u^{l}}{l !}=\sum_{q, l=0}^{\infty}{ }_{H} F_{q+l}(w, y ; z) \frac{t^{q}}{q !} \frac{u^{l}}{l !} .
$$

Now replacing $q$ by $q-n, l$ by $l-p$ and using the lemma ([11, p.100(1)]):

$$
\sum_{k=0}^{\infty} \sum_{n=0}^{\infty} A(n, k)=\sum_{k=0}^{\infty} \sum_{n=0}^{k} A(n, k-n),
$$

in the 1.h.s. of (3.6), we find

$$
\begin{gathered}
\sum_{q, l=0}^{\infty} \sum_{n, p=0}^{q, l} \frac{(w-x)^{n+p}}{n ! p !}{ }_{H} F_{q+l-n-p}(x, y ; z) \frac{t^{q}}{(q-n) !} \frac{u^{l}}{(l-p) !} \\
=\sum_{q, l=0}^{\infty}{ }_{H} F_{q+l}(w, y ; z) \frac{t^{q}}{q !} \frac{u^{l}}{l !} .
\end{gathered}
$$

Finally, on equating the coefficients of the like powers of $t$ and $u$ in the above equation, we get the assertion (3.1) of Theorem 3.1.

Remark 3.1. Taking $l=0$ in assertion (3.1) of Theorem 3.1, we deduce the following consequence of Theorem 3.1 .

Corollary 3.1. The following summation formula for Hermite-Fubini polynomials ${ }_{H} F_{n}(x, y ; z)$ holds true:

$$
{ }_{H} F_{q}(w, y ; z)=\sum_{n=0}^{q}\left(\begin{array}{l}
q \\
n
\end{array}\right)(w-x)^{n}{ }_{H} F_{q-n}(x, y ; z) .
$$

Remark 3.2. Replacing $w$ by $w+x$ in (3.9), we obtain

$$
{ }_{H} F_{q}(x+w, y ; z)=\sum_{n=0}^{q}\left(\begin{array}{c}
q \\
n
\end{array}\right) w^{n}{ }_{H} F_{q-n}(x, y ; z) .
$$

Theorem 3.2. The following summation formula for Hermite-Fubini polynomials ${ }_{H} F_{n}(x, y ; z)$ holds true:

$$
\begin{gathered}
{ }_{H} F_{n}(w, u ; z){ }_{H} F_{m}(W, U ; Z)=\sum_{r, k=0}^{n, m}\left(\begin{array}{c}
n \\
r
\end{array}\right)\left(\begin{array}{c}
m \\
k
\end{array}\right) H_{r}(w-x, u-y)_{H} F_{n-r}(x, y ; z) \\
\times H_{k}(W-X, U-Y)_{H} F_{m-k}(X, Y ; Z)
\end{gathered}
$$


Proof. Consider the product of the Hermite-Fubini polynomials, we can be written as generating function (2.1) in the following form:

$\frac{1}{1-z\left(e^{t}-1\right)} e^{x t+y t^{2}} \frac{1}{1-Z\left(e^{T}-1\right)} e^{X T+Y T^{2}}=\sum_{n=0}^{\infty}{ }_{H} F_{n}(x, y ; z) \frac{t^{n}}{n !} \sum_{m=0}^{\infty}{ }_{H} F_{m}(X, Y ; Z) \frac{T^{m}}{m !}$.

Replacing $x$ by $w, y$ by $u, X$ by $W$ and $Y$ by $U$ in (3.12) and equating the resultant to itself,

$$
\begin{gathered}
\sum_{n=0}^{\infty} \sum_{m=0}^{\infty}{ }_{H} F_{n}(w, u ; z){ }_{H} F_{m}(W, U ; Z) \frac{t^{n}}{n !} \frac{T^{m}}{m !} \\
=\exp \left((w-x) t+(u-y) t^{2}\right) \exp \left((W-X) T+(U-Y) T^{2}\right) \\
\times \sum_{n=0}^{\infty} \sum_{m=0}^{\infty}{ }_{H} F_{n}(x, y ; z)_{H} F_{m}(X, Y ; Z) \frac{t^{n}}{n !} \frac{T^{m}}{m !},
\end{gathered}
$$

which on using the generating function (3.7) in the exponential on the r.h.s., becomes

$$
\begin{gathered}
\sum_{n=0}^{\infty} \sum_{m=0}^{\infty}{ }_{H} F_{n}(w, u ; z)_{H} F_{m}(W, U ; Z) \frac{t^{n}}{n !} \frac{T^{m}}{m !} \\
=\sum_{n, r=0}^{\infty} H_{r}(w-x, u-y)_{H} F_{n}(x, y ; z) \frac{t^{n+r}}{n ! r !} \sum_{m, k=0}^{\infty} H_{k}(W-X, U-Y)_{H} F_{m}(X, Y ; Z) \frac{T^{m+k}}{m ! k !} .
\end{gathered}
$$

Finally, replacing $n$ by $n-r$ and $m$ by $m-k$ and using equation (3.7) in the r.h.s. of the above equation and then equating the coefficients of like powers of $t$ and $T$, we get assertion (3.11) of Theorem 3.2.

Remark 3.3. Replacing $u$ by $y$ and $U$ by $Y$ in assertion (3.11) of Theorem 3.2, we deduce the the following consequence of Theorem 3.2.

Corollary 3.2. The following summation formula for Hermite-Fubini polynomials ${ }_{H} F_{n}(x, y ; z)$ holds true:

$$
\begin{gathered}
{ }_{H} F_{n}(w, u ; z)_{H} F_{m}(W, U ; Z)=\sum_{r, k=0}^{n, m}\left(\begin{array}{c}
n \\
r
\end{array}\right)\left(\begin{array}{c}
m \\
k
\end{array}\right)(w-x)^{r}{ }_{H} F_{n-r}(x, y ; z) \\
\times(W-X)^{k}{ }_{H} F_{m-k}(X, Y ; Z)
\end{gathered}
$$

Theorem 3.3. The following summation formula for Hermite-Fubini polynomials ${ }_{H} F_{n}(x, y ; z)$ holds true:

$$
{ }_{H} F_{n}(x+w, y+u ; z)=\sum_{s=0}^{n}\left(\begin{array}{c}
n \\
s
\end{array}\right){ }_{H} F_{n-s}(x, y ; z) H_{s}(w, u) .
$$

Proof. We replace $x$ by $x+w$ and $y$ by $y+u$ in (2.1), use (1.2) and rewrite the generating function as:

$$
\begin{gathered}
\frac{1}{1-z\left(e^{t}-1\right)} \exp \left((x+w) t+(y+u) t^{2}\right)=\sum_{n=0}^{\infty}{ }_{H} F_{n}(x, y ; z) \frac{t^{n}}{n !} \sum_{s=0}^{\infty} H_{s}(w, u) \frac{t^{s}}{s !} \\
=\sum_{n=0}^{\infty} H_{n} F_{n}(x+w, y+u ; z) \frac{t^{n}}{n !}
\end{gathered}
$$


Now replacing $n$ by $n-s$ in l.h.s. and comparing the coefficients of $t^{n}$ on both sides, we get the result (3.15).

Theorem 3.4. The following summation formula for Hermite-Fubini polynomials ${ }_{H} F_{n}(x, y ; z)$ holds true:

$$
{ }_{H} F_{n}(y, x ; z)=\sum_{s=0}^{\left[\frac{n}{2}\right]} F_{n-2 s}(y ; z) \frac{x^{s}}{(n-2 s) ! s !} .
$$

Proof. We replace $x$ by $y$ and $y$ by $x$ in equation (2.1) to get

$$
\sum_{n=0}^{\infty}{ }_{H} F_{n}(y, x ; z) \frac{t^{n}}{n !}=\sum_{n=0}^{\infty} F_{n}(y ; z) \frac{t^{n}}{n !} \sum_{s=0}^{\infty} \frac{x^{s} t^{2 s}}{k !} .
$$

Now replacing $n$ by $n-2 s$ in r.h.s. and comparing the coefficients of $t$ on both sides, we arrive at the desired result (3.16).

Theorem 3.5. The following summation formula for Hermite-Fubini polynomials ${ }_{H} F_{n}(x, y ; z)$ holds true:

$$
{ }_{H} F_{n}(x, y ; z)=\sum_{r=0}^{n}\left(\begin{array}{c}
n \\
r
\end{array}\right) F_{n-r}(x-w ; z) H_{r}(w, y) .
$$

Proof. By exploiting the generating function (1.2), we can write equation (2.1) as

$$
\frac{1}{1-z\left(e^{t}-1\right)} e^{(x-w) t} e^{w t+y t^{2}}=\sum_{n=0}^{\infty} F_{n}(x-w ; z) \frac{t^{n}}{n !} \sum_{r=0}^{\infty} H_{r}(w, y) \frac{t^{r}}{r !} .
$$

On replacing $n$ by $n-r$ in above equation, we get

$$
\sum_{n=0}^{\infty}{ }_{H} F_{n}(x, y ; z) \frac{t^{n}}{n !}=\sum_{n=0}^{\infty} \sum_{r=0}^{n} F_{n-r}(x-w ; z) H_{r}(w, y) \frac{t^{n}}{(n-r) ! r !} .
$$

Equating the coefficients of the like powers of $t$ on both sides, we get (3.19).

Theorem 3.6. The following summation formula for Hermite-Fubini polynomials ${ }_{H} F_{n}(x, y ; z)$ holds true:

$$
{ }_{H} F_{n}(x+1, y ; z)=\sum_{r=0}^{n}\left(\begin{array}{c}
n \\
r
\end{array}\right){ }_{H} F_{n-r}(x, y ; z) .
$$

Proof. Using the generating function (2.1), we have

$$
\begin{gathered}
\sum_{n=0}^{\infty}{ }_{H} F_{n}(x+1, y ; z) \frac{t^{n}}{n !}-\sum_{n=0}^{\infty}{ }_{H} F_{n}(x, y ; z) \frac{t^{n}}{n !} \\
=\left(\frac{1}{1-z\left(e^{t}-1\right)}\right)\left(e^{t}-1\right) e^{x t+y t^{2}} \\
=\sum_{n=0}^{\infty}{ }_{H} F_{n}(x, y ; z) \frac{t^{n}}{n !}\left(\sum_{r=0}^{\infty} \frac{t^{r}}{r !}-1\right) \\
=\sum_{n=0}^{\infty}{ }_{H} F_{n}(x, y ; z) \frac{t^{n}}{n !} \sum_{r=0}^{\infty} \frac{t^{r}}{r !}-\sum_{n=0}^{\infty}{ }_{H} F_{n}(x, y ; z) \frac{t^{n}}{n !} \\
=\sum_{n=0}^{\infty} \sum_{r=0}^{n}\left(\begin{array}{c}
n \\
r
\end{array}\right){ }_{H} F_{n-r}(x, y ; z) \frac{t^{n}}{n !}-\sum_{n=0}^{\infty}{ }_{H} F_{n}(x, y ; z) \frac{t^{n}}{n !} .
\end{gathered}
$$


Finally, equating the coefficients of the like powers of $t$ on both sides, we get $(3.21)$.

\section{Symmetric identities}

Recently, Khan [7], Pathan and Khan [9, 10] have been introduced symmetric identities. In this section, we establish general symmetry identities for the generalized Hermite-Fubini polynomials ${ }_{H} F_{n}(x, y ; z)$ by applying the generating function (2.1) and (2.2).

Theorem 4.1. Let $x, y, z \in \mathbb{R}$ and $n \geq 0$, then the following identity holds true:

$$
\begin{aligned}
& \sum_{r=0}^{n}\left(\begin{array}{c}
n \\
r
\end{array}\right) b^{r} a_{H}^{n-r} F_{n-r}\left(b x, b^{2} y ; z\right)_{H} F_{r}\left(a x, a^{2} y ; z\right) \\
= & \sum_{r=0}^{n}\left(\begin{array}{c}
n \\
r
\end{array}\right) a^{r} b_{H}^{n-r} F_{n-r}\left(a x, a^{2} y ; z\right)_{H} F_{r}\left(b x, b^{2} y ; z\right) .
\end{aligned}
$$

Proof. Start with

$$
A(t)=\frac{1}{\left(1-z\left(e^{a t}-1\right)\right)\left(1-z\left(e^{b t}-1\right)\right)} e^{a b x t+a^{2} b^{2} y t^{2}} .
$$

Then the expression for $A(t)$ is symmetric in $a$ and $b$ and we can expand $A(t)$ into series in two ways to obtain:

$$
\begin{gathered}
A(t)=\sum_{n=0}^{\infty}{ }_{H} F_{n}\left(b x, b^{2} y ; z\right) \frac{(a t)^{n}}{n !} \sum_{r=0}^{\infty}{ }_{H} F_{r}\left(a x, a^{2} y ; z\right) \frac{(b t)^{r}}{r !} \\
A(t)=\sum_{n=0}^{\infty}\left(\sum_{r=0}^{n}\left(\begin{array}{c}
n \\
r
\end{array}\right) b^{r} a_{H}^{n-r} F_{n-r}\left(b x, b^{2} y ; z\right)_{H} F_{r}\left(a x, a^{2} y ; z\right)\right) \frac{t^{n}}{n !} .
\end{gathered}
$$

Similarly, we can show that

$$
\begin{gathered}
A(t)=\sum_{n=0}^{\infty}{ }_{H} F_{n}\left(a x, a^{2} y ; z\right) \frac{(b t)^{n}}{n !} \sum_{r=0}^{\infty} H_{r} F_{r}\left(b x, b^{2} y ; z\right) \frac{(a t)^{r}}{r !} \\
A(t)=\sum_{n=0}^{\infty}\left(\sum_{r=0}^{n}\left(\begin{array}{c}
n \\
r
\end{array}\right) a^{r} b^{n-r}{ }_{H} F_{n-r}\left(a x, a^{2} y ; z\right)_{H} F_{r}\left(b x, b^{2} y ; z\right)\right) \frac{t^{n}}{n !} .
\end{gathered}
$$

By comparing the coefficients of $\frac{t^{n}}{n !}$ on the right hand sides of the last two equations, we arrive at the desired result (4.1).

Theorem 4.2. For each pair of integers $a$ and $b$ and all integers and $n \geq 0$, the following identity holds true:

$$
\begin{aligned}
& \sum_{k=0}^{n}\left(\begin{array}{c}
n \\
k
\end{array}\right) \sum_{i=0}^{a-1} \sum_{j=0}^{b-1} a^{n-k} b_{H}^{k} F_{n-k}\left(b x+\frac{b}{a} i+j, b^{2} y, z\right) F_{k}(a u, z) \\
& \sum_{k=0}^{n}\left(\begin{array}{c}
n \\
k
\end{array}\right) \sum_{j=0}^{a-1} \sum_{i=0}^{b-1} b^{n-k} a_{H}^{k} F_{n-k}\left(a x+\frac{a}{b} i+j, a^{2} y, z\right) F_{k}(b u, z) .
\end{aligned}
$$

Proof. Let

$$
B(t)=\frac{e^{a b(x+u) t+a^{2} b^{2} y t^{2}}\left(e^{a b t}-1\right)^{2}}{\left(1-z\left(e^{a t}-1\right)\right)\left(1-z\left(e^{b t}-1\right)\right)\left(e^{a t}-1\right)\left(e^{b t}-1\right)}
$$




$$
\begin{gathered}
=\frac{e^{a b x t+a^{2} b^{2} y t^{2}}}{1-z\left(e^{a t}-1\right)} \frac{e^{a b t}-1}{e^{b t}-1} \frac{e^{a b u t}}{1-z\left(e^{b t}-1\right)} \frac{e^{a b t}-1}{e^{a t}-1} \\
B(t)=\frac{e^{a b x t+a^{2} b^{2} y t^{2}}}{1-z\left(e^{a t}-1\right)} \sum_{i=0}^{a-1} e^{b t i} \frac{e^{a b u t}}{1-z\left(e^{b t}-1\right)} \sum_{j=0}^{b-1} e^{a t j} \\
=\frac{e^{a^{2} b^{2} y t^{2}}}{1-z\left(e^{a t}-1\right)} \sum_{i=0}^{a-1} \sum_{j=0}^{b-1} e^{\left(b x+\frac{b}{a} i+j\right) a t} \sum_{k=0}^{\infty} F_{k}(a u, z) \frac{(b t)^{k}}{k !} \\
=\sum_{n=0}^{\infty}\left(\sum_{k=0}^{n}\left(\begin{array}{c}
n \\
k
\end{array}\right) \sum_{i=0}^{a-1} \sum_{j=0}^{b-1} a^{n-k} b^{k} F_{n-k}\left(b x+\frac{b}{a} i+j, b^{2} y, z\right) F_{k}(a u, z)\right) \frac{t^{n}}{n !}
\end{gathered}
$$

On the other hand

$$
B(t)=\sum_{n=0}^{\infty}\left(\sum_{k=0}^{n}\left(\begin{array}{c}
n \\
k
\end{array}\right) \sum_{j=0}^{a-1} \sum_{i=0}^{b-1} b^{n-k} a_{H}^{k} F_{n-k}\left(a x+\frac{a}{b} i+j, a^{2} y, z\right) F_{k}(b u, z)\right) \frac{t^{n}}{n !} .
$$

By comparing the coefficients of $t^{n}$ on the right hand sides of the last two equations, we arrive at the desired result.

\section{References}

[1] Bell, E. T, Exponential polynomials, Ann. of Math., 35(1934), 258-277.

[2] Boyadzhiev, K. N, A series transformation formula and related polynomials, Int. J. Math. Math. Sci., 23(2005), 3849-3866.

[3] Dattoli, G, Lorenzutta, S and Cesarano, C, Finite sums and generalized forms of Bernoulli polynomials, Rendiconti di Mathematica, 19(1999), 385-391.

[4] Dil, A, Kurt, V, Investing geometric and exponential polynomials with EulerSeidel matrices, J. Integer Sequences, 14(2011), 1-12.

[5] Graham, R. L, Knuth, D. E, Patashnik, O, Concrete Mathematics, AddisonWesley Publ. Co., New York, 1994.

[6] Gross, O. A, Preferential arrangements, Amer. Math. Monthly, 69(1962), 4-8.

[7] Khan, W. A, Some properties of the generalized Apostol type Hermite-Based polynomials, Kyungpook Math. J., 55(2015), 597-614.

[8] Kargin, L, Some formulae for products of Fubini polynomials with applications, arXiv:1701.01023v1[math.CA] 23 Dec 2016.

[9] Pathan, M. A and Khan, W. A, Some implicit summation formulas and symmetric identities for the generalized Hermite-Bernoulli polynomials, Mediterr. J. Math., 12(2015), 679-695.

[10] Pathan, M. A and Khan, W. A: A new class of generalized polynomials associated with Hermite and Euler polynomials, Mediterr. J. Math., 13(2016), 913-928.

[11] Srivastava, H. M and Manocha, H. L, A treatise on generating functions, Ellis Horwood Limited. Co., New York, 1984.

[12] Tanny, S. M, On some numbers related to Bell numbers, Canad. Math. Bull., 17(1974), 733-738. 